\title{
Model approach of a robotic manipulator 3DOF using Machine Learning
}

\author{
Darielson A. Souza*, Josias G. Batista*, Laurinda L. N. dos Reis*, and Antônio Barbosa de Souza Júnior ${ }^{\dagger}$ \\ *Robotics, Automation and Control Research Group (GPAR), \\ Federal University of Ceará, Fortaleza, CE, Brazil ; \\ ${ }^{\dagger}$ Federal Institute of Ceara - IFCE Campus Maracanau, Maracanau, CE, Brazi \\ darielson@dee.ufc.br, josiasgb@dee.ufc.br, laurinda@dee.ufc.br, barbosa@dee.ufc.br
}

\begin{abstract}
Applications of robotics have been steadily expanding in recent years, and robotics is evolving every day. Currently, robotics is seen as an important area in many applications. Robotics and computational intelligence are increasingly working in parallel with the goal of better performance and productivity. This work has the objective of making an modeling of a robotic arm with three phase induction motor through machine learning techniques to obtain a better model that represents the plant. The techniques used were Artificial Neural Network (ANNs): MLP and ELM. The techniques obtained a good performance, and they were evaluated through the multi-correlation coefficient $\left(R^{2}\right)$ for a comparative analysis.
\end{abstract}

Index Terms-model approach, MLP ANN, ELM ANN, robot manipulator

\section{INTRODUCTION}

The area of robotic systems is very promising, and is quite relevant in many applications. Control techniques have been constantly researched and tested in robotics 1. High performance control techniques are of paramount importance for successful tasks in robotics, such as for example in industrial robotic manipulators which perform many repetitive trajectories. The main characteristics of manipulator robots in industries are: speed, torque and precision, these attributes are essential for a smooth operation 2].

One of the characteristics of a robotic manipulator in general is the presence of a claw at the end of its link, and is designed for components to be transported taking into account the characteristics of the material, such as weight and shape, and must be adjusted to handle such objects. The [3] makes the study of a fuzzy modeling of the dynamics of the system that is developed with data generated by a hydrogen fuel cell simulator.

The proposed method will be compared with other identification approaches, such as MLP(Multilayer perceptron) ANN and ELM(Extreme learning machine) ANN. The methods are compared and evaluated by the multiple correlation coefficient $\left(R^{2}\right)$, where the transfer functions generated by each method will be tested.

This work is structured by sections, the first is the introduction which presents an overview on the work and a contextualization. Section II will show the technical details of the robotic manipulator, which was used. Section III presents the system modeling methods used. Section IV shows the results and discussions, containing charts and tables to be evaluated and compared. Finally, V demonstrates the conclusions about the results and can be a contribution to other works that may arise.

\section{Technical Details}

In this work, a cylindircal robot manipulator is used. The cylindrical manipulator has 5 degrees of freedom (DOF), the first DOF is the base that rotates, the 2nd DOF is linear that is the trunk that makes the movements in the vertical direction, the third DOF is what makes the movements in the horizontal, and the last DOFs: fourth and fifth DOF is the claw that is also called the end effector [4]. Fig. 1 presents a manipulator used in the work where in its joint the actuators are three-phase induction motors. The drive of the manipulator it was a Texas Instruments TMS320F2812 microcontroller digital signal processor (DSP) is used. The main advantage of it is that in addition to high performance it has the ability to perform 150 MPIS (millions of instructions per second), the intrinsic support to vector spatial modulation, SVPWM [5].

The remaining instruments are a Hall-effect current sensors, auxiliary voltage sources, a three-phase voltage inverter module by Semikron with a switching frequency of $2.5 \mathrm{kHz}$, a multi-turn precision potentiometer coupled to the motor shaft, with a sampling time of $0.4 \mathrm{~ms}$. The structure is driven by a three-phase induction motor (IM) squirrel cage type. The power of the motor was chosen so that it was possible to move the structure of the manipulator. The movement transmission to the manipulator's first degree of freedom occurs through the use of belt and pulleys. For powering the circuits and plates, an auxiliary voltage source capable of providing 4 levels of continuous voltage was developed. Being $18 \mathrm{~V}$ the voltage for switching the triggers of the three-phase inverter, $15 \mathrm{~V}$ and $-15 \mathrm{~V}$ the voltage for the current sensors and $5 V$ the voltage for the board power of signal conditioning. 


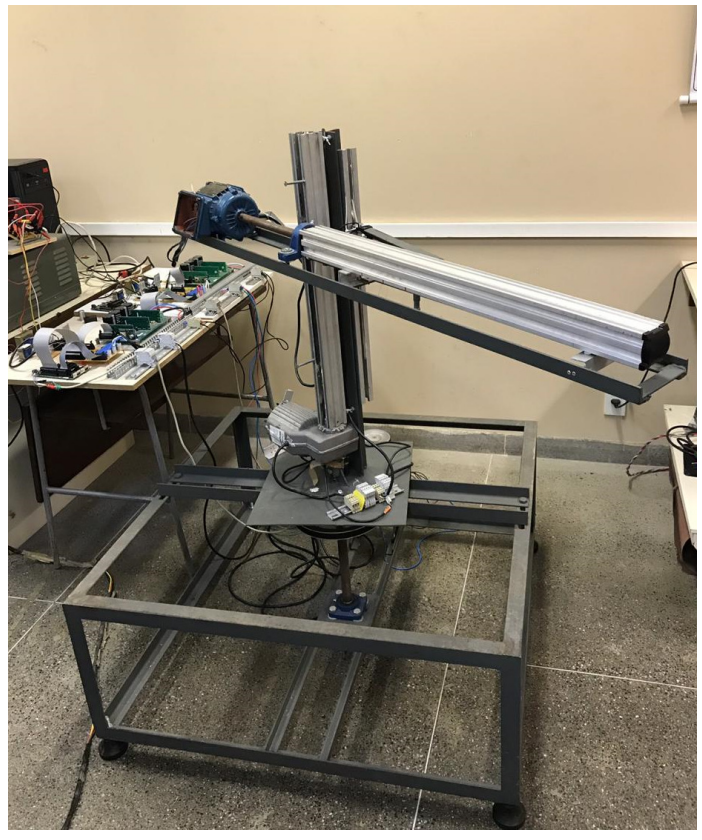

Fig. 1. Robotic manipulator.

\section{System Modeling}

According to [6], the identification process is defined in an approximation of models having a real model as a reference. Some identification steps are done by shuffling the database, separating the training labels and a part for testing.

Most of the processes are nonlinear and time-varying. However, linear models are often employed to model and control such processes. As operational conditions change, it is important that the model and its characteristics be used for benefits of advanced model-based control [6]. It is therefore interesting to make an adequate formulation of the algorithms to provide efficient identification procedures. This work present a ANNs: MLP and ELM.

\section{A. MLP ANN}

One of the major advantages of Artificial Neural Networks is its ability to learn in representations of linear and nonlinear models. The most common type of neural network to solve nonlinear separation problems is the MLP neural network which stands for Multi Layer Perceptron. This type of ANNs is a super-network, since it requires a type of output wanted to learn [7. The purpose of this network is to create a model that can correctly map input and output, even when the desired output is unknown. Figure 2 shows an ANN MLP.

ANN MLP can learn using a kind of training called backpropation, which means backpropagation, input data is presented repeatedly to the network. In each iteration the output is compared to the desired output and an error is calculated, this error is fed into the network for weight

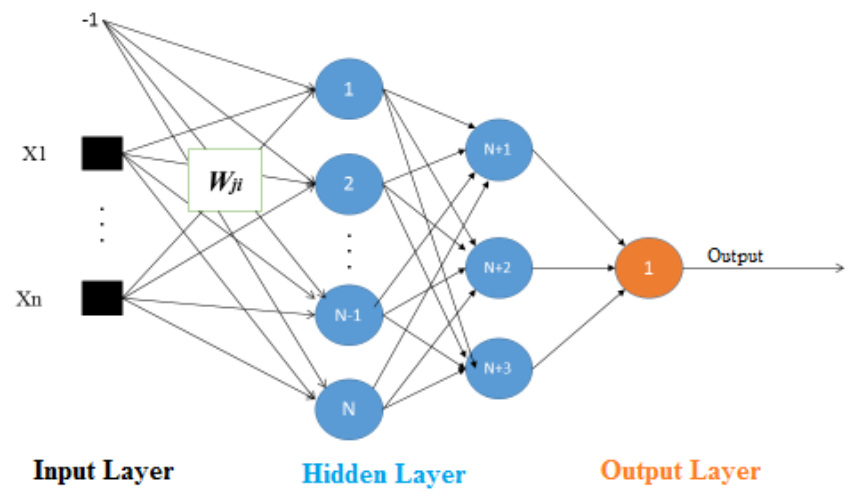

Fig. 2. Architecture of ANN MLP

adjustments in the way that this error is minimized in each round until the network approaches the desired result.

ANN MLP can learn using a kind of training called backpropation, which means backpropagation, input data is presented repeatedly to the network. In each iteration the output is compared to the desired output and an error is calculated, this error is fed into the network for weight adjustments in the way that this error is minimized in each round until the network approaches the desired result. Below is an algorithm based on neural network MLP.

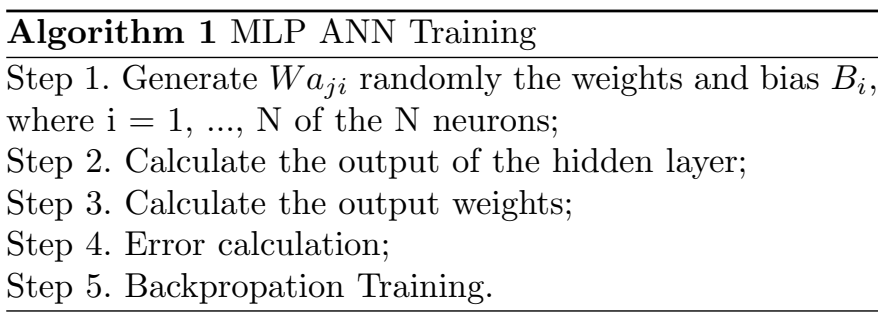

\section{B. ELM ANN}

The extreme learning machines are feedforward neural networks for sorting, regression, clustering, sparse approximation, compaction, and resource learning with a single layer or multiple layers of hidden neurons, where hidden neuron parameters (not just weights connecting inputs to us hidden) need not be tuned. These hidden nodes can be ran-domly assigned and never updated (that is, they are random projections but with non-linear transfor-mations) or they can be inherited from their ancestors without being altered. In most cases, the output weights of hidden nodes are usually learned in a single step, which is basically equivalent to learning a linear model [8].

Some works the ELM network was used to solve problems of reverse kinetics in other types of robots like: 10 The ELM network has several advantages over the others, such as: the input weights and the polari-zation of the hidden layer are chosen randomly, another advantage is that the weights of the output layer are determined analytically 9 . In the following algorithm a step-by-step of the network will be shown. 
Algorithm 2 ELM Training

Step 1. Select randomly values for the weights $w_{j i}$ and bias $b_{j i}, \mathrm{i}=1, \ldots, \mathrm{N}$;

Step 2. Calculate the output matrix $H$ of the hidden layer;

Step 3. Calculate the output weights.

Where:

$w_{j i}$ : Are the synaptic weights

$b_{j i}:$ It's bias

$H$ : Hidden layer output

Several architectures were used for ANN with the vari ation of some facets, thus obtaining the expected results The ELM RNA was the only algorithm that had 4 dat. inputs per bias account. The inputs input data were th Cartesian coordinates, and the output $\mathrm{Y}$ : are the trajec tory points of each joint (motor). Regarding the faces tha were fixed with the best results were: number of neuron in the hidden layer equals 6 , the learning step was 0.6 , th weight ranges were -0.001 to 1 .

\section{Results}

To illustrate the effectiveness of the proposed algo rithms, simulations and experiments are carried out baser on a 3-DOF manipulator robot arm as shown in Fig. 1. I] this work only the data acquisition of the 3rd degree wa obtained and the mechanical and electrical behavior wa collected to obtain the identification of the current an velocity during the activation of the 1st joint. The dat. obtained for current and velocity when was used a step response with different amplitudes conform presented in 5]. These data represent the behavior of the 1st joint what was used in proposed identification method. In this case the current is taken as input and the velocity is taken as output, Fig. 3 .
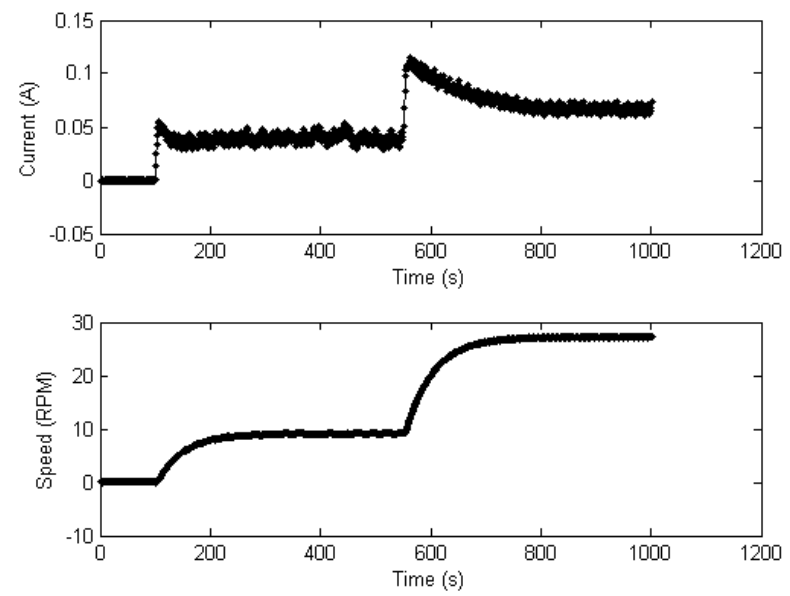

Fig. 3. Input and output data.

The methods MLP and ELM are compared. To furthe validate the parameter estimation performance, the correlation index, $R^{2}$ of the estimated parameters is evaluated and the computational cost. It is important to compare with classical methods, since the validation of these will prove the effectiveness of the research.

The models presented have already been validated, where the results of the complete dataset are shown.

Figure 4 shows the approximation of the model using the MLP network, where the final configuration was 1 hidden layer and 20 neurons, learning step of 0.7 and 300 epoch quantities.

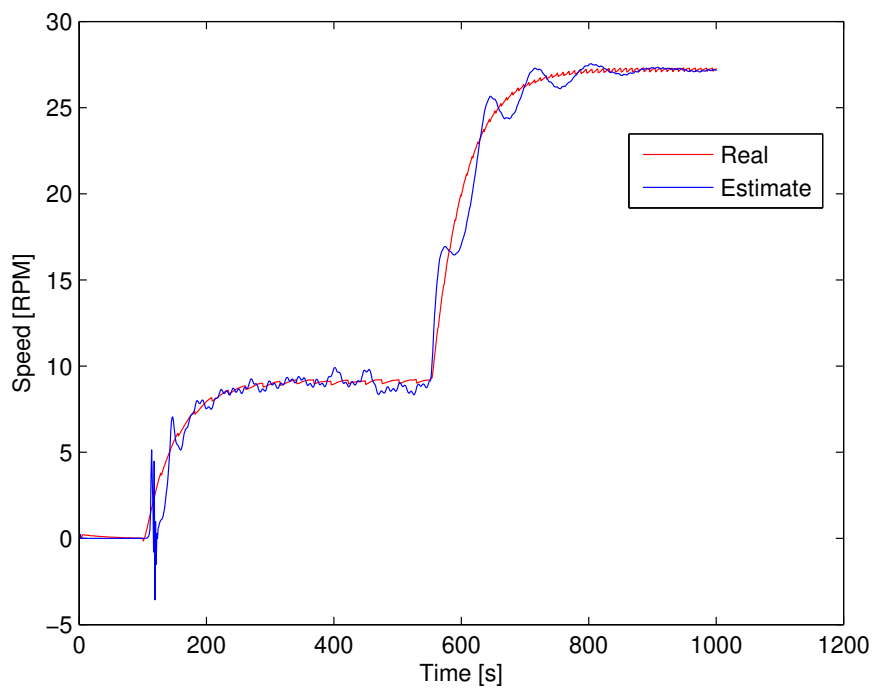

Fig. 4. MLP ANN

Figure 5 shows the results of ELm modeling. The method obtained a good performance, however the importance of using this technique as a way to make a comparative analysis very competitive.

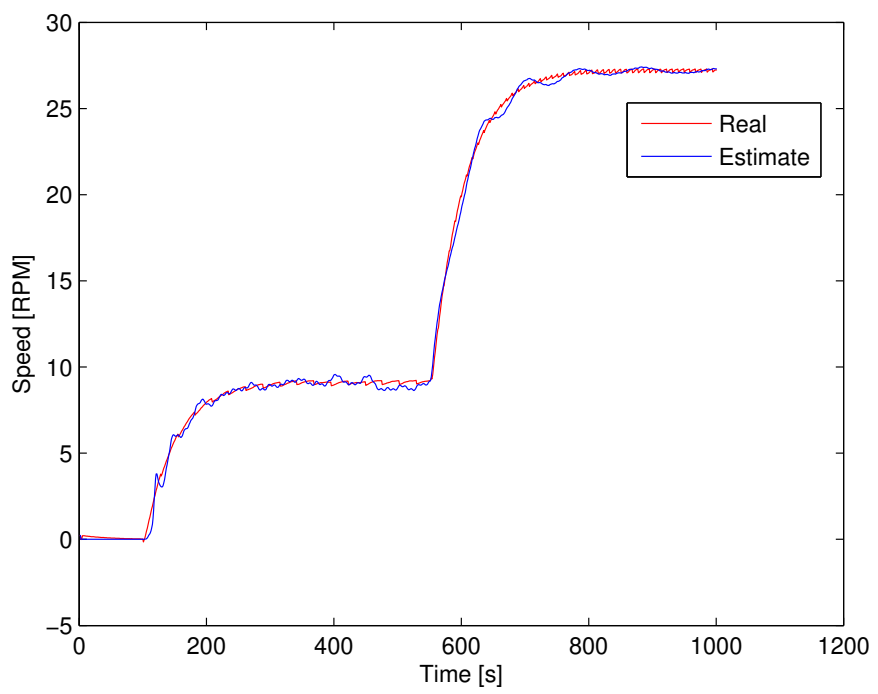

Fig. 5. ELM ANN 
It can be observed from the figures that the ELM modeling system achieved a better result in comparison with the MLP ANN.

Table I shows the index $R^{2}$ results and the computational cost of each method. The $R^{2}$ is used to evaluate the quality of the estimate.

TABLE I

Comparison of $R^{2}$ AND Cost Results

\begin{tabular}{rll}
\hline Methods & $R^{2}$ & Computational cost $[\mathrm{s}]$ \\
\hline$M L M$ & 0.8123 & 29.2 \\
$E L M$ & 0.9141 & 5.1 \\
\hline
\end{tabular}

According to the table $\mathbb{I}$ and the presented results, the ELM ANN obtained better results in comparison to the other methods tested. The ELM ANN was able to training the parameters weigth, consequently the results improved significantly.

\section{Conclusions}

The work presented methods of modeling parameters of one of the robotic manipulator joints. The technique proposed to be compared with other proposed, achieved a better performance, which was a Extreme learning machine Artificial Neural Network. The classical methods that were used is: Multilayer perceptron. The ELM ANN technique performed better when compared to MLP ANN.

\section{REFERENCES}

[1] Pinto, Milena F. et al. Modified approach using variable charges to solve inherent limitations of potential fields method. In: 2014 11th IEEE/IAS International Conference on Industry Applications. IEEE, 2014.

[2] Alemany, Ahmed; Shang, Helen. Recursive sub-space identification with prior information using the constrained least squares approach. Computers Chemical Engineering, v. 54, p. 174-180, 2013.

[3] Bertone, Ana Maria Amarillo, Jefferson Beethoven Martins, and Keiji Yamanaka, Black-box fuzzy identification of a nonlinear hydrogen fuel cell model, TEMA (São Carlos) 18.3 (2017): 405418.

[4] L. R. Rebouças; C. G. M. Filho; A. B. S. Junior; L. L. N. Reis; F. G. Nogueira; T. R. F. Neto; L. H. Barreto; B. C. Torrico. Predictive Control Applied in 3-phase Squirrel Cage Induction Motor for Zero Speed, SBAI, 2017.

[5] L. R. Rebouças; C. G. M. Filho; A. B. S. Junior; L. L. N. Reis; T. R. F. Neto; L. H. Barreto. Identificação de um motor de indução trifásico aplicado para um Manipulador Robotico. 2015.

[6] A, A. R. Coelho.; L, S. Colho. Identificação de sistemas Dinâmicos Lineares, Editora UFSC, $2^{\mathrm{O}}$ Edição, 2015.

[7] HAYKIN, S.(2001). Neural Networks: Principios and Pratice (in Portuguese), Bookman, ed.

[8] HUANG, GUANG-BIN; ZHU, QIN-YU; SIEW, CheeKheong. Extreme learning machine: theory and applications. Neurocomputing., 2006.

[9] HUANG, GUANG-BIN, QIN-YU ZHU, K. Z. MAO; CHEEKHEONG SIEW, P. SARATCHANDRAN, AND N. SUNDARARAJAN. Can Threshold Networks Be Trained Directly?. IEEE Transactions on Circuits and Systems. 2006.

[10] ZHIYU ZHOU. Inverse kinematics solution for robotic manipulator based on extreme learning machine and sequential mutation genetic algorithm. International Journal of Advanced Robotic Systems, 2018. 